\title{
The role of LIM and SH3 protein-1 in bladder cancer metastasis
}

\author{
MISAKI SATO $^{1}$, MIHOKO SUTOH YONEYAMA ${ }^{1,2}$, SHINGO HATAKEYAMA ${ }^{2}$, TOMIHISA FUNYU ${ }^{1}$, \\ TADASHI SUZUKI ${ }^{1}$, CHIKARA OHYAMA ${ }^{2}$ and SHIGERU TSUBOI ${ }^{1,2}$ \\ ${ }^{1}$ Department of Cancer Immunology and Cell Biology, Oyokyo Kidney Research Institute, Hirosaki, Aomori 036-8243; \\ ${ }^{2}$ Department of Urology, Hirosaki University Graduate School of Medicine, Hirosaki, Aomori 036-8562, Japan
}

Received April 19, 2017; Accepted August 4, 2017

DOI: $10.3892 / 01.2017 .6802$

\begin{abstract}
The LIM and SH3 protein-1 (LASP-1) is a multi-domain protein that is involved in several malignant cancers. The role of LASP-1 in malignant phenotypes including high invasive properties and unrestricted cell proliferation, remain to be elucidated. The present study reported the association of LASP-1 expression with bladder cancer malignancy and its role in cancer cell invasion and proliferation. The immunohistochemical analysis of the expression status of LASP-1 in radical cystectomy specimens from invasive bladder cancer patients revealed that the LASP-1-positive patients demonstrated a decreased survival rate compared with the LASP-1-negative patients. The expression level of LASP-1 was increased in invasive bladder cancer cell lines compared with the non-invasive bladder cancer cell lines. Invasive cancer cells form invadopodia, the filamentous actin-based membrane protrusions that are essential in cancer cell invasion. Knockdown of LASP-1 reduced the ability to form invadopodia, resulting in decreased invasive capacity of the LASP-1 knockdown cells. In addition, knockdown of LASP-1 reduced cell proliferation. These results suggest that LASP-1 is important in invadopodia formation and cell proliferation of bladder cancer cells, promoting the malignant properties and resulting in poor-prognosis.
\end{abstract}

\section{Introduction}

Bladder cancer is one of the most prevalent urologic malignancy and the fourth common cancer among men worldwide (1). There are several treatment choices for bladder cancer patients including radical cystectomy, radiation therapy and prospective instillation of chemotherapy and immunotherapy $(2,3)$. Although the treatment of bladder cancer has been improved in recent years, it frequently recurs due to metastasis and its

Correspondence to: Dr Shigeru Tsuboi, Department of Cancer Immunology and Cell Biology, Oyokyo Kidney Research Institute, 90 Kozawa Yamazaki, Hirosaki, Aomori 036-8243, Japan

E-mail: tsuboi@oyokyo.jp

Key words: LIM and SH3 protein, LASP-1, bladder cancer, metastasis, invadopodia prognosis with 5-year overall survival rate are unsatisfactory (4). Therefore, it is urgently necessary to elucidate the molecular mechanism of bladder cancer metastasis to discover novel biomarkers for the further improvement of bladder cancer treatment.

Among the multiple processes involved in bladder cancer metastasis, cancer cell invasion is considered a key process. For cancer cell invasion process, highly invasive cancer cells form invadopodia, the filamentous actin (F-actin)-based membrane protrusions to remodel and degrade extracellular matrix (ECM) and invade surrounding tissues (5). Although invadopodia are considered to play important roles in the steps of the metastatic cascade, the details of the invadopodia formation have not been fully understood yet.

LIM and SH3 protein-1 (LASP-1) is a multi-domain protein. The LASP-1 protein contains a LIM (Lin-11, IsI1, MEC-3) protein-protein interaction domain (6,7), two F-actin-binding nebulin (NEBU) domains and Src homology (SH) 3 domain and it was shown that LASP-1 regulates cytoskeleton dynamics $(8,9)$. Several groups reported that overexpression of LASP-1 has been observed in a variety of malignant tumors including metastatic breast cancer (10), ovarian cancer (11) and medulloblastoma (12). Recent clinical studies have demonstrated that LASP is one of the useful biomarkers for bladder cancer detection $(13,14)$ and that LASP-1 is involved in bladder cancer metastasis (15). The above studies on the LASP-1 function and its high expression in malignant bladder cancer led us to postulate that LASP-1 is involved in bladder cancer malignant phenotypes such as cell invasion through the regulation of actin cytoskeleton.

In the present study, we found the correlation of LASP-1 expression with clinicopathological characteristics in bladder cancer and also performed functional analysis to determine the biological functions of LASP-1 in cancer metastasis.

\section{Materials and methods}

Cells, reagents and antibodies. Four established human bladder cancer cell lines were used. KK-47 was a generous gift of Dr. T. Matsuo (Tohoku University, Sendai, Japan) (16). RT-4 and T24 were purchased from American Type Culture Collection. A human invasive and high-grade bladder cancer cell line, YTS-1 was a generous gift of Dr Kakizaki H. (Yamagata University, Yamagata, Japan) (17). Cells were maintained in RPMI-1640 medium (Sigma-Aldrich; Merck KGaA, Darmstadt, Germany) 
supplemented with $10 \%$ fetal bovine serum (FBS; Capricorn Scientific $\mathrm{GmbH}$, Ebsdorfergrund, Germany) with $5 \% \mathrm{CO}_{2}$ at $37^{\circ} \mathrm{C}$. The monoclonal antibodies to LASP-1 (clone 86C) and glyceraldehyde-3-phospahte dehydrogenase (GAPDH) (clone 3H12) were purchased from Abcam (Cambridge, UK) and MBL (Woburn, MA, USA), respectively.

Analyses of bladder cancer patients. The cohort consisted of 48 patients with muscle invasive bladder cancer treated with radical cystectomy at the Department of Urology, Hirosaki University Graduate School of Medicine (Hirosaki, Japan). Bladder cancer specimens from patients were fixed with $10 \%$ buffered formalin for 12-24 h. Paraffin-embedded lymph node samples were cut at $3 \mu \mathrm{m}$ and subjected to hematoxylin and eosin staining and immunohistochemistry using a monoclonal antibody to LASP-1. Anti-mouse immunoglobulin antibody conjugated with horseradish peroxidase (Agilent Technologies Japan, Ltd., Tokyo, Japan) was used as a secondary antibody and peroxidase activity was visualized with Liquid $\mathrm{DAB}^{+}$Substrate Chromogen System (Agilent Technologies Japan, Ltd.). Based on the histochemical staining results, specimens that showed LASP-1-positive in greater than $50 \%$ of cancer cells were judged as LASP-1-positive. Patient survival was compared to the results of immunohistochemistry results. The institutional ethics committee of Hirosaki University Graduate School of Medicine approved this study.

LASP-1 knockdown (LASPKD). YTS-1 cells with reduced expression of LASP-1 (LASPKD cells) were generated by shRNA technology as previously described (18). An shRNA expression plasmid was constructed using pBAsi-hU6 Neo DNA (Takara Bio, Inc., Otsu, Japan). The shRNA sequence for LASP-1 was: GATCCAAGGTGAACTGTCTGGATA AGCTGTGAAGCCACAGATGGGCTTATCCAGACAGTT CACCTTTTTTTTA (The siRNA sequence for LASP-1 was underlined.) (19). A human non-targeting siRNA sequence (Accell siRNA control; Thermo Fisher Scientific, Inc., Waltham, MA, USA) was used to prepare the control cells expressing non-targeting shRNA. We transfected cells with LASP-1 shRNA expression plasmid together with pEGFP-C2 vector (BD Biosciences, Palo Alto, CA, USA) and pTK-Hyg Vector (Clontech Laboratories, Inc., Mountain View, CA, USA) using Lipofectamine ${ }^{\circledR} 2000$ (Invitrogen; Thermo Fisher Scientific, Inc.). EGFP-positive cells were separated using BD FACSAria (BD Biosciences, Franklin Lakes, NJ, USA) and then cultured in RPMI-1640 medium containing $10 \% \mathrm{FBS}$ and $50 \mu \mathrm{g} / \mathrm{ml}$ hygromycin B for 2-4 days. Greater than $70 \%$ of cultured cells were positive for EGFP.

Western blotting. Total lysates of cancer cells were prepared by solubilization in $50 \mathrm{mM}$ Tris- $\mathrm{HCl}$ buffer, $\mathrm{pH} 7.5$, containing 1\% Igepal CA-630, $150 \mathrm{mM} \mathrm{NaCl}$ and proteinase inhibitors. The lysates were resolved by SDS-PAGE on an 8-16\% gradient gel (Invitrogen; Thermo Fisher Scientific, Inc.), and transferred to polyvinylidene fluoride (PVDF) membrane. Western blot analyses were performed using the specific primary antibodies and a horseradish peroxidase-conjugated secondary antibody. Signals were visualized using the ECL PLUS detection system (GE Healthcare Life Sciences, Little Chalfont, UK).
Immunofluorescence microscopy. Cells seeded on coverslips were fixed in $4 \%$ paraformaldehyde and permeabilized with PBS containing $0.1 \%$ saponin and $1 \%$ bovine serum albumin (BSA). Cells were stained with Alexa Fluor 568-labelled phalloidin (Invitrogen; Thermo Fisher Scientific, Inc.) together with the monoclonal antibody to LASP-1, and Alexa Fluor 488-labelled secondary antibody (Invitrogen; Thermo Fisher Scientific, Inc.) was used for antibody staining. Cell staining was examined under an Olympus IX-73 fluorescence microscope (Olympus Corp., Tokyo, Japan).

Matrigel invasion assay. Invasion assay was performed using a conventional Transwell system (BD Biosciences, San Jose, CA, USA). The bottom surface of the filter (with $8 \mu \mathrm{m}$ pore size) of the upper chamber was coated with $100 \mu \mathrm{g} / \mathrm{ml}$ of fibronectin and the top surface was covered with $1 \mathrm{mg} / \mathrm{ml}$ of Matrigel Matrix (BD Biosciences). The lower chamber was filled with RPMI-1640 medium supplemented with FBS. EGFP-positive bladder cancer cells $\left(5 \times 10^{4}\right)$ were placed into the upper chamber. After incubation at $37^{\circ} \mathrm{C}$ for $24 \mathrm{~h}$, non-migrated cells remaining at the top surface of the filter were carefully removed with cotton swabs. Migrated cells on the bottom surface were fixed with $4 \%$ paraformaldehyde and counted under a fluorescence microscope (Olympus IX-73).

Proliferation assay. For cancer cell proliferation assay, cells were seeded in 96-well plates $\left(1 \times 10^{4}\right.$ cells per well) and cultured at $37^{\circ} \mathrm{C}$ for indicated time. Cell growth was assayed using Cell Counting Kit-8 (Dojindo Laboratories, Kumamoto, Japan) according to the manufacturer's specification.

Statistical analysis. We used the statistical program SPSS 12.0 (SPSS, Inc., Chicago, IL, USA). Statistically significant differences were determined using the Student's t-test. $\mathrm{P}<0.05$ was considered to indicate a statistically significant difference.

\section{Results}

Expression of LASP-1 in bladder cancer tissues. The domain organization of LASP-1 (Fig. 1A) showed that LASP-1 contains the N-terminal LIM domain, two F-actin binding domain (NEBU domain) and C-terminal SH3 domain. To evaluate the importance of LASP-1 in prognosis of bladder cancer patients, we immunohistochemically examined the expression status of LASP-1 in radical cystectomy specimens from 48 bladder cancer patients using anti-LASP-1 monoclonal antibody. The typical LASP-1-positive staining pattern showed LASP-1 in the cytoplasm (Fig. 1B). Based on the staining status, the patients were divided into two groups, those with LAPS-1-positive specimens (Fig. 1B) and those with C2GnT-negative specimens (Fig. 1C). A Kaplan-Meier analysis showed that LASP-1-positive patients $(n=33)$ survived for shorter time than the LASP-1-negative patients $(n=15)$ (Fig. 1D). The factor that has the strongest impact on prognosis is recurrence due to metastasis. These results suggest that bladder cancer highly expressing LASP-1 was malignant and metastatic.

Increased expression of LASP-1 in invasive bladder cancer cells. To explore what roles LASP-1 plays in malignant 
A

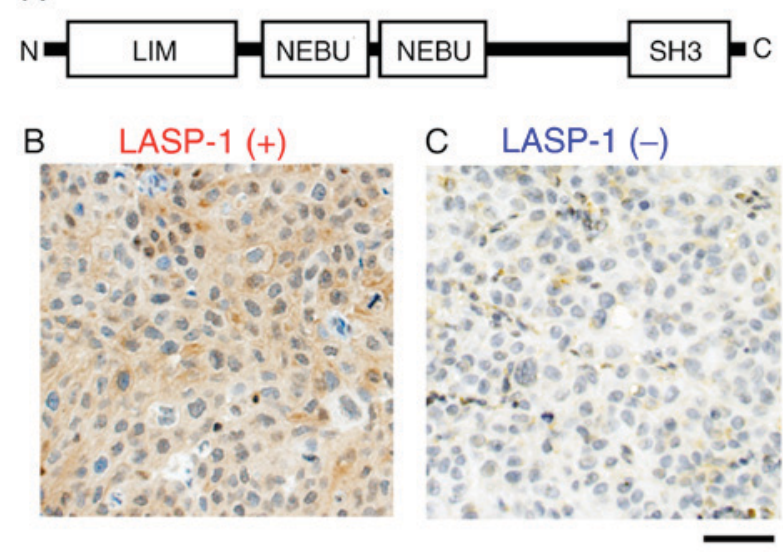

D

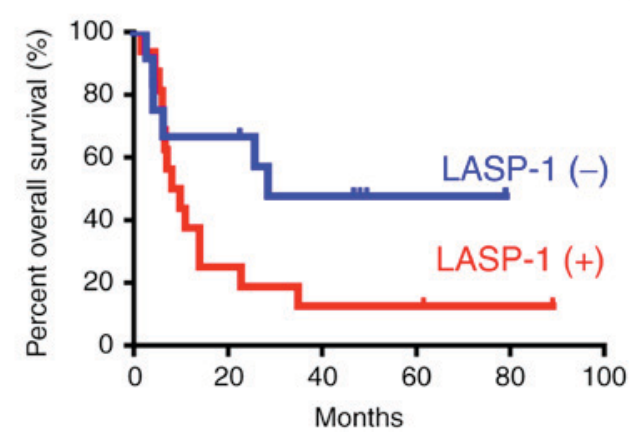

Figure 1. Expression of LIM and SH3 protein-1 (LASP-1) in bladder cancer. (A) Domain organization of LASP-1. LASP-1 contains a LIM domain, two NEBU domains and SH3 domain. (B and C) Immunohistochemistry of bladder tumor specimens using anti-LASP-1 monoclonal antibody. (B) LASP-1 positive tumor. LASP-1 staining of a LASP-1 positive tumor specimen exhibited a typical cytoplasmic pattern. Bar, $50 \mu \mathrm{m}$. (C) LASP-1 negative tumor. (D) Kaplan-Meier curve for the cause-specific survival of bladder cancer patients according to LASP-1 staining status. A total of 48 bladder cancer patients were divided into two groups according to the expression status of LASP-1, LASP-1 positive ( $n=33$, red line) and LASP-1 negative $(n=15$, blue line).

bladder cancer cells, we first examine the expression level of LASP-1 in bladder cancer cell lines. The expression level of LASP-1 was increased in invasive bladder cancer cell lines, T24 and YTS-1 (Fig. 2A, lanes 3 and 4), compared with the non-invasive bladder cancer cell lines, RT-4 and KK-47 (Fig. 2A, lanes 1 and 2). This suggests that LASP-1 is involved in bladder cancer cell invasion. To further examine the roles of LASP-1 in cancer cell invasion, we established LASPKD cells by silencing the expression of LASP-1 in YTS-1 (an invasive bladder cancer cell line) cells using shRNA technology. Western blotting showed that LASP-1 expression was reduced in LASPKD cells (Fig. 2B, lane 2) compared with control cells (Fig. 2B, lane 1).

Reduction of invadopodia formation in LASPKD cells. We previously reported that invadopodia formation is critical for the invasion capacity of bladder cancer cells (20-22). We hypothesized that higher expression of LASP-1 in bladder cancer cells contributes to highly invasive property by regulating invadopodia formation. Invadopodia are the membrane protrusions enriched by polymerized filamentous actin (F-actin). YTS-1 cells which are invasive bladder cancer cells were permeabilized and stained with Alexa Fluor 568-labeled phalloidin to visualize F-actin cores of invadopodia. Phalloidin
A
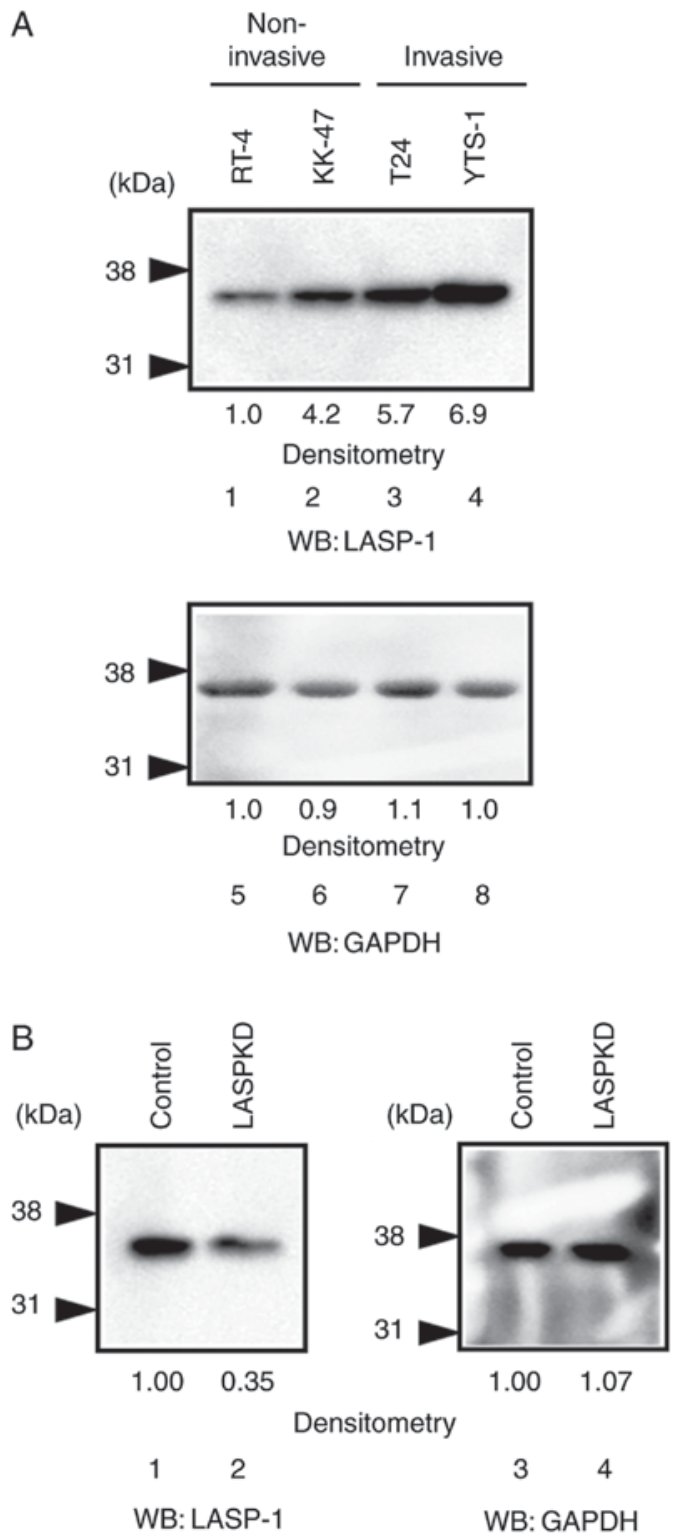

Figure 2. Expression level of LIM and SH3 protein-1 (LASP-1) in bladder cancer cells. (A) Western blotting for the expression levels of LASP-1 (upper panel) and GAPDH (lower panel) in bladder cancer cells. Four established bladder cancer cell lines were used: the non-invasive bladder cancer cells, RT-4 and KK-47 and the invasive bladder cancer cells, T24 and YTS-1. Numbers below blots indicate the relative band intensities quantified by a densitometer. (B) Establishment of LASP-1 knockdown bladder cancer cells. Expression level of LASP-1 was reduced in LASP-1 knockdown cells. Total protein samples prepared form YTS-1 control (Control) and LASP-1 knockdown YTS-1 cells were analyzed by western blotting for the expression of LASP-1 and GAPDH [LASP-1 knockdown (LASPKD)]. Numbers below blots indicate the relative band intensities quantified by a densitometer in both (A and B).

staining of YTS-1 cells gives lots of F-actin puncta (Fig. 3A). These punctate structures are typical invadopodia (Several typical ones were denoted by arrows). To determine the role of LASP-1 in invadopodia formation, we examined the invadopodia formation in LASPKD cells. YTS-1 cells expressing human non-targeting siRNA were examined as a control cell (Control). Control cells exhibited a typical cytosolic staining of LASP-1 (Fig. 3B) and the expression of LASP-1 was reduced in LASPKD cells (Fig. 3D). Numerous invadopodia were observed in control cells (Fig. 3C; several typical ones 
A

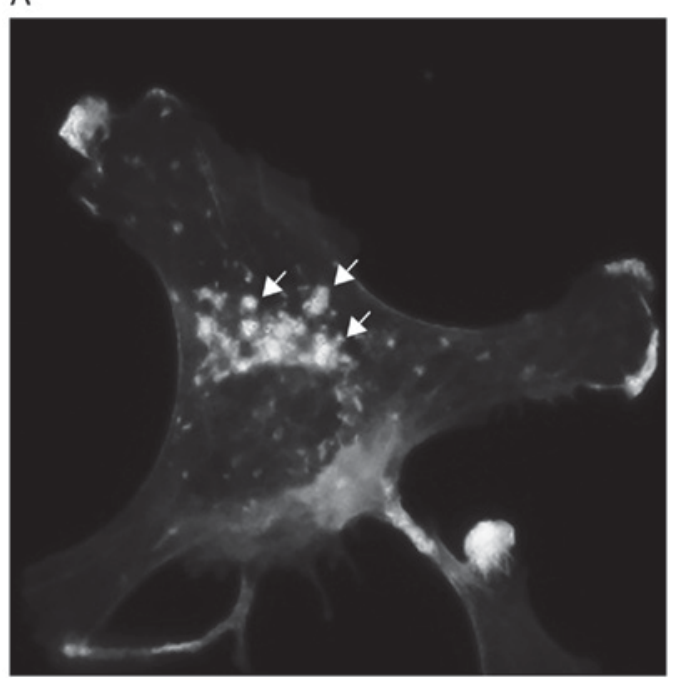

$\mathrm{F}$

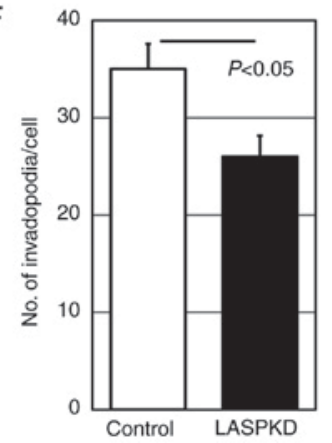

G
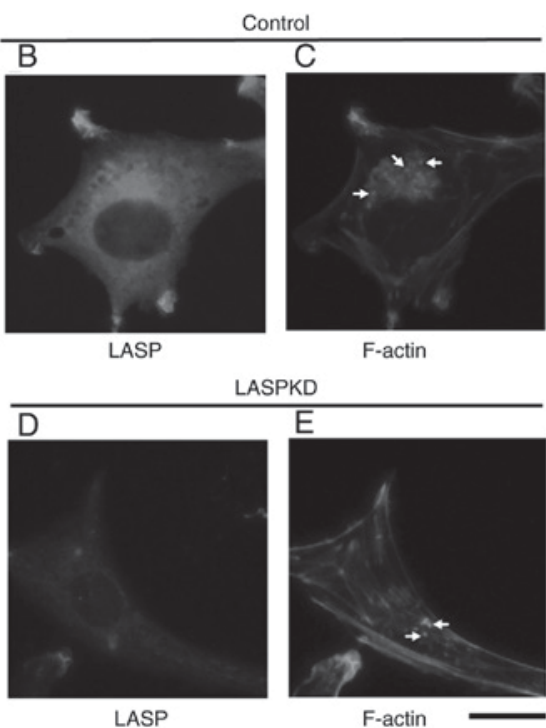

LASP

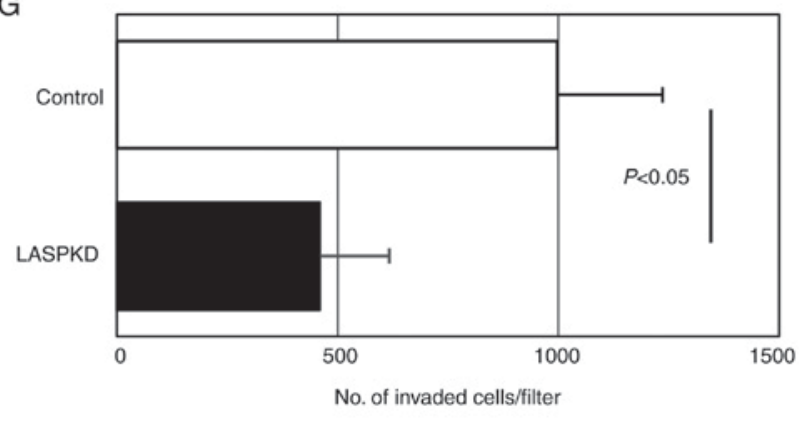

Figure 3. Reduced formation of invadopodia formation in LIM and SH3 protein-1 (LASP-1) knockdown bladder cancer cells. (A) Invadopodia formation in an invasive bladder cancer cell line, YTS-1. Cells were permeabilized and stained with Alexa Fluor 568-labelled phalloidin to visualize the F-actin cores of invadopodia. Several typical invadopodia were denoted by arrows. Bar, $20 \mu \mathrm{m}$. (B-F) Reduced invadopodia formation in LASP-1 knockdown cells (LASPKD). Cells were double-stained with (B and D) anti-LASP-1 monoclonal antibody and (C and E) Alexa Fluor 568-labeled phalloidin. (B and C) Immunofluorescence micrographs of YTS control cell (Control) and (D and E) LASPKD. Several typical invadopodia were denoted by arrows. Bar, $20 \mu \mathrm{m}$. (F) The number of invadopodia formed in YTS control cell (Control) and LASPKD were counted for 20 cells. Data represent mean \pm standard error (SE) of three independent experiments. (G) Matrigel invasion capacity of YTS control cells (Control) and LASPKD was measured by a conventional invasion assay using a Boyden chamber. Data represent mean $\pm \mathrm{SE}$ of three independent experiments.

were denoted by arrows), which was equivalent to YTS-1 cell (Fig. 3A). Although LASPKD cells had an ability to form invadopodia, the number of invadopodia was significantly decreased compared with control cells (denoted by arrows) (Fig. 3E and F). Invadopodia play a critical role in cancer cell invasion. We next examined in vitro invasive capacity of LASPKD cells by using a conventional invasion assay method in a Boyden chamber. Invasive capacity of LASPKD cells was significantly lower than that of control cells (Fig. 3G). These results indicate that LASPKD cells exhibit less invasive capacity due to the reduced number of invadopodia, suggesting that LASP-1 play an important role in invasion by promoting invadopodia formation.

Reduced cell proliferation in LASPKD cells. Another important biological process in which LASP-1 may be involved is cell proliferation. To examine the impact of LASP-1 on cellular proliferative ability, we monitored the cell proliferation in LASPKD cells for four consecutive days. LASPKD cells exhibited a marked reduction of cell proliferation compared with control cells (Fig. 4), suggesting that LASP-1 expression is critical to cell proliferation.

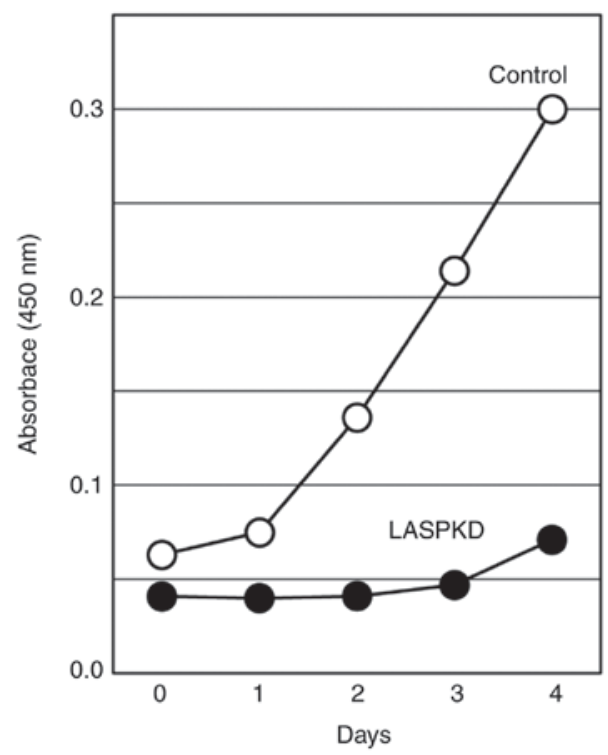

Figure 4. Proliferation of LASP-1 knockdown (LASPKD) cells. To determine the effect of reduced expression of LASP-1 on cancer cell proliferation, cell growth of LASPKD cells was compared with that of YTS control cells (Control) for 4 days. LASP-1, LIM and SH3 protein-1. 


\section{Discussion}

We observed a high expression of LASP-1 in malignant bladder cancer specimens compared with benign specimens (Fig. 1B). We also showed that higher LASP-1 expression correlated with poor prognosis (Fig. 1C). LASP-1 was originally identified from a cDNA library of metastatic breast cancer cells (23). In addition, there was a study reporting that LASP-1 expression was significantly higher in oral squamous cell carcinoma than that in normal oral tissues (24). Our results taken together with these previous observations suggest that higher expression of LASP-1 is involved in the development of metastatic phenotype. This is supported by the recent observation that LASP-1 can be a promising urinary marker for detection of bladder cancer $(13,14)$.

One of the malignant phenotypes of cancer which mediate metastasis is invasion. Higher invasion capacity leads to metastasis. In order for cancer cells to efficiently invade surrounding tissues, invadopodia formation is required (25-27). Our previous studies showed that bladder cancer cells forming invadopodia exhibited the high capacity of transurothelial invasion through the bladder muscle layers and extravasation from the blood vessels, resulting in metastasis $(22,28)$. This study showed that LASP-1 is necessary for invadopodia formation and Matrigel invasion (Fig. 3). These results suggest that LASP-1 plays a critical role in metastasis by promoting invadopodia formation. This is supported by a recent study reporting LASP-1 regulates the function of podosomes in macrophages. Podosomes are the F-actin-based membrane structures which are homologous with invadopodia (19). Future investigation into the detailed mechanisms of the involvement of LASP-1 in the formation of invadopodia and podosomes should be required.

Unrestricted cell proliferation is one of the hallmarks of malignant cancers. Our result indicates that LASP-1 silencing results in a strong inhibition of cancer cell proliferation (Fig. 4). This suggests that LASP-1 plays an essential role in cell proliferation. LASP-1 is involved in cell cycle $\mathrm{G} 2 / \mathrm{M}$ phase transition of oral cancer cells (24). Our result taken together with this observation strongly suggests that overexpression of LASP-1 increases cell proliferation in bladder cancer cells, promoting its malignancy.

In conclusion, our data suggest that LASP-1 plays the important roles in both invadopodia formation and cell proliferation, contributing to the promotion of malignant phenotypes of poor-prognosis bladder cancer. It is noteworthy that LASP-1 may be a useful biomarker of bladder cancer and a possible therapeutic target for developing anti-cancer drugs because of its importance in cancer malignant phenotypes such as high invasiveness and unrestricted cell proliferation.

\section{Acknowledgements}

This study was supported by the grants-in-aid from the Mizutani Foundation (S.T.) and Japanese Society for the Promotion of Science (15K06989) (S.T.), (grant nos. 15H02563, 15K15579 and 25220206) (C.O.).

\section{References}

1. Siegel RL, Miller KD and Jemal A: Cancer statistics, 2016. CA Cancer J Clin 66: 7-30, 2016.
2. Weintraub MD, Li QQ and Agarwal PK: Advances in intravesical therapy for the treatment of non-muscle invasive bladder cancer (Review). Mol Clin Oncol 2: 656-660, 2014.

3. Booth CM, Siemens DR, Li G, Peng Y, Tannock IF, Kong W, Berman DM and Mackillop WJ: Perioperative chemotherapy for muscle-invasive bladder cancer: A population-based outcomes study. Cancer 120: 1630-1638, 2014.

4. Sfakianos JP, Kim PH, Hakimi AA and Herr HW: The effect of restaging transurethral resection on recurrence and progression rates in patients with nonmuscle invasive bladder cancer treated with intravesical bacillus Calmette-Guérin. J Urol 191: 341-345, 2014.

5. Even-Ram S and Yamada KM: Cell migration in 3D matrix. Curr Opin Cell Biol 17: 524-532, 2005.

6. Way JC and Chalfie M: mec-3, a homeobox-containing gene that specifies differentiation of the touch receptor neurons in $\mathrm{C}$. Elegans. Cell 54: 5-16, 1988.

7. Karlsson S and Ahrén B: Insulin and glucagon secretion in swimming mice: Effects of autonomic receptor antagonism. Metabolism 39: 724-732, 1990.

8. Chew CS, Parente JA Jr, Zhou C, Baranco E and Chen X: Lasp-1 is a regulated phosphoprotein within the cAMP signaling pathway in the gastric parietal cell. Am J Physiol 275: C56-C67, 1998.

9. Grunewald TG and Butt E: The LIM and SH3 domain protein family: Structural proteins or signal transducers or both? Mol Cancer 7: 31, 2008.

10. Grunewald TG, Kammerer U, Schulze E, Schindler D, Honig A, Zimmer M and Butt E: Silencing of LASP-1 influences zyxin localization, inhibits proliferation and reduces migration in breast cancer cells. Exp Cell Res 312: 974-982, 2006.

11. Grunewald TG, Kammerer U, Winkler C, Schindler D, Sickmann A, Honig A and Butt E: Overexpression of LASP-1 mediates migration and proliferation of human ovarian cancer cells and influences zyxin localisation. Br J Cancer 96: 296-305, 2007.

12. Traenka C, Remke M, Korshunov A, Bender S, Hielscher T, Northcott PA, Witt H, Ryzhova M, Felsberg J, Benner A, et al: Role of LIM and SH3 protein 1 (LASP1) in the metastatic dissemination of medulloblastoma. Cancer Res 70: 8003-8014, 2010.

13. Ardelt P, Grunemay N, Strehl A, Jilg C, Miernik A, Kneitz B and Butt E: LASP-1, a novel urinary marker for detection of bladder cancer. Urol Oncol 31: 1591-1598, 2013.

14. Payton S: Bladder cancer: LASP-1-a promising urine marker for detection of bladder cancer. Nat Rev Urol 9: 240, 2012.

15. Li Z, Chen Y, Wang X, Zhang H, Zhang Y, Gao Y, Weng M, Wang L, Liang H, Li M, et al: LASP-1 induces proliferation, metastasis and cell cycle arrest at the G2/M phase in gallbladder cancer by down-regulating S100P via the PI3K/AKT pathway. Cancer Lett 372: 239-250, 2016.

16. Kubota Y, Nakada T, Yanai H, Kakizaki H, Sasagawa I and Watanabe M: Electropermeabilization in bladder cancer chemotherapy. Cancer Chemother Pharmacol 39: 67-70, 1996.

17. Hisazumi H, Uchibayashi T, Katoh M, Kobayashi T, Nakajima K, Naitoh K, Misaki T and Kuroda K: Anticancer drug sensitivity in vitro in the bladder cancer cell line, KK-47 and prophylactic use of carbazilquinone and urokinase in bladder cancer. Urol Res 9: 231-235, 1981.

18. Tsuboi S, Sutoh M, Hatakeyama S, Hiraoka N, Habuchi T, Horikawa Y, Hashimoto Y, Yoneyama T, Mori K, Koie T, et al: A novel strategy for evasion of NK cell immunity by tumours expressing core2 O-glycans. EMBO J 30: 3173-3185, 2011.

19. Stölting M, Wiesner C, van Vliet V, Butt E, Pavenstädt H, Linder S and Kremerskothen J: Lasp-1 regulates podosome function. PLoS One 7: e35340, 2012.

20. Sutoh M, Hashimoto Y, Yoneyama T, Yamamoto $H$, Hatakeyama S, Koie T, Okamoto A, Yamaya K, Saitoh H, Funyu T, et al: Invadopodia formation by bladder tumor cells. Oncol Res 19: 85-92, 2010.

21. Yamamoto H, Sutoh M, Hatakeyama S, Hashimoto Y, Yoneyama T, Koie T, Saitoh H, Yamaya K, Funyu T, Nakamura T, et al: Requirement for FBP17 in invadopodia formation by invasive bladder tumor cells. J Urol 185: 1930-1938, 2011.

22. Sutoh Yoneyama M, Hatakeyama S, Habuchi T, Inoue T, Nakamura T, Funyu T, Wiche G, Ohyama C and Tsuboi S: Vimentin intermediate filament and plectin provide a scaffold for invadopodia, facilitating cancer cell invasion and extravasation for metastasis. Eur J Cell Biol 93: 157-169, 2014. 
23. Tomasetto C, Moog-Lutz C, Regnier CH, Schreiber V, Basset $\mathrm{P}$ and Rio MC: Lasp-1 (MLN 50) defines a new LIM protein subfamily characterized by the association of LIM and SH3 domains. FEBS Lett 373: 245-249, 1995.

24. Shimizu F, Shiiba M, Ogawara K, Kimura R, Minakawa Y Baba T, Yokota S, Nakashima D, Higo M, Kasamatsu A, et al: Overexpression of LIM and SH3 Protein 1 leading to accelerated $\mathrm{G} 2 / \mathrm{M}$ phase transition contributes to enhanced tumourigenesis in oral cancer. PLoS One 8: e83187, 2013.

25. Caldieri G, Ayala I, Attanasio F and Buccione R: Cell and molecular biology of invadopodia. Int Rev Cell Mol Biol 275: 1-34, 2009.
26. Linder S, Wiesner $\mathrm{C}$ and Himmel M: Degrading devices: Invadosomes in proteolytic cell invasion. Annu Rev Cell Dev Biol 27: 185-211, 2011.

27. Murphy DA and Courtneidge SA: The 'ins' and 'outs' of podosomes and invadopodia: Characteristics, formation and function. Nat Rev Mol Cell Biol 12: 413-426, 2011.

28. Imanishi K, Yoneyama MS, Hatakeyama S, Yamamoto $H$, Koie T, Saitoh H, Yamaya K, Funyu T, Nakamura T, Ohyama C and Tsuboi S: Invadopodia are essential in transurothelial invasion during the muscle invasion of bladder cancer cells. Mol Med Rep 9: 2159-2165, 2014. 